\title{
Clinical and Radiological Evaluation After Chemical Synovectomy With Rifampicin in Hemophilic Arthropathy: Korean Experience With a 2-Week Interval Protocol
}

\author{
Hoon Chang Suh, $\mathrm{MD}^{1}$, Don-Kyu Kim, MD, PhD ${ }^{1}$, Si Hyun Kang, MD, PhD ${ }^{1}$, \\ Kyung Mook Seo, MD, $\mathrm{PhD}^{1}$, Hee Sang Kim, $\mathrm{MD}^{1}$, Ji Young Lee, $\mathrm{MD}^{1}$, \\ Sang Yoon Lee, $\mathrm{MD}, \mathrm{PhD}^{2}$, Ki Young Yoo, $\mathrm{MD}^{3}$
}

\begin{abstract}
${ }^{1}$ Department of Physical Medicine and Rehabilitation, Chung-Ang University, College of Medicine, Seoul; ${ }^{2}$ Department of Rehabilitation Medicine, Seoul National University Boramae Medical Center, Seoul; ${ }^{3}$ Korea Hemophilia Foundation, Seoul, Korea
\end{abstract}

Objective To assess the clinical outcome of chemical synovectomy with rifampicin in hemophilic arthropathy by using the World Federation of Hemophilia (WFH) scoring system and plain radiograph.

Methods We performed rifampicin synovectomy (RS) on 30 joints of 28 hemophilic patients diagnosed as hemophilic arthropathy stage I-III (based on Fernandez-Palazzi clinical classification). Clinical status (bleeding frequency, pain, joint physical status) and radiological staging were evaluated as parts of the WFH scoring system before and 1 year after RS. The patients were divided into two groups by the Arnold-Hilgartner scale of the initial $\mathrm{X}$-ray as stage 3 or less for the low-stage group $(\mathrm{n}=17)$ and over 3 for the high-stage group $(\mathrm{n}=13)$.

Results Total WFH joint physical scores were reduced after injection, and the number of bleeding episodes and pain showed especially significant improvement. For other subscores of the WFH joint physical score, only swelling, range of motion, and crepitus showed statistically significant improvement. According to the severity of the radiologic finding, the WFH joint physical score of both the low-stage and high-stage groups showed significant improvement. In the radiological aspect, the low-stage group, without joint space narrowing at the initial plain radiograph, showed no further aggravation after injection. However, in the high-stage group, radiology found aggravation regardless of the procedure.

Conclusion It is suggested that chemical synovectomy with rifampicin may prevent hemarthrosis and improve clinical symptoms. Especially in the early stage of arthropathy without joint-space narrowing, it seems to have an additional benefit that delays radiological aggravation and preserves joint status.

Keywords Hemophilia, Arthropathy, Rifampicin, Synovectomy

Received May 16, 2017; Accepted September 4, 2017

Corresponding author: Don-Kyu Kim

Department of Physical Medicine and Rehabilitation, Chung-Ang University Hospital, Chung-Ang University College of Medicine, 102 Heukseok-ro, Dongjak-gu, Seoul 06973, Korea. Tel: +82-2-6299-1881, Fax: +82-2-6298-1866, E-mail: donkim21@cau.ac.kr

ORCID: Hoon Chang Suh (http://orcid.org/0000-0001-5333-8986); Don-Kyu Kim (http://orcid.org/0000-0001-8619-830X); Si Hyun Kang (http://orcid. org/0000-0003-0205-067X); Kyung Mook Seo (http://orcid.org/0000-0002-9762-085X); Hee Sang Kim (http://orcid.org/0000-0002-8759-1725); Ji Young Lee (http://orcid.org/0000-0002-2291-8790); Sang Yoon Lee (http://orcid.org/0000-0002-2906-3094); Ki Young Yoo (http://orcid.org/0000-0003-1470-3519).

(c) This is an open-access article distributed under the terms of the Creative Commons Attribution Non-Commercial License (http://creativecommons.org/ licenses/by-nc/4.0) which permits unrestricted noncommercial use, distribution, and reproduction in any medium, provided the original work is properly cited. Copyright (C) 2018 by Korean Academy of Rehabilitation Medicine 


\section{INTRODUCTION}

In hemophilia, intra-articular hemorrhage (haemarthroses) is the most common site of serious bleeding. Recurrent bleeding finally leads to chronic arthropathy, up to $86.67 \%$ [1]. The most frequently affected joints are the elbows, knees, and ankles [2]. Once haemarthroses occurred, the collected blood-breakdown products act as potent stimulants to the synovium inducing inflammation and damage the metabolism of chondrocytes [3]. Repeated bleedings stimulate synovial inflammation and progress to synovial hypertrophy and developing vascular synovium. In this chronic synovitis stage, the vicious cycle leads to progressive joint degeneration and destruction $[4,5]$. The primary aim of care for haemarthroses in hemophilia is to prevent and treat bleeding by supplementing the deficient clotting factor [6]. The Medical and Scientific Advisory Council of the National Hemophilia Foundation, the World Federation of Hemophilia (WFH), and the World Health Organization have recommended prophylaxis (defined as treatment by intravenous injection of factor concentrate in anticipation of and in order to prevent bleeding) to prevent the arthropathic complications of hemophilia [6-8]. However, despite aggressive regimens of factor-replacement therapy, breakthrough bleeding and subclinical bleeding also occur, leading to structural damage of joints [4]. A chronic stage of synovitis can cause chronic pain, swelling, contracture, limb deformity, and limitation of motion. Thus, by causing hemarthrosis, synovitis leads to functional, psychiatric, and socio-economic problems and deterioration of the quality of life [9]. Hemophilia is an untreatable condition, and planning for lifelong care is essential to prevent intra-articular bleeding and chronic synovitis.

During the phase of chronic synovitis with recurrent bleeding, surgical synovectomy has proven effective in reducing the frequency of bleeding $[5,10,11]$. RodriguezMerchan [2] have reported good results of $85 \%$ in rifampicin and yttrium-90 radiation synovectomy as compared to $70 \%$ in surgical synovectomy. However, because it is a surgical procedure under general anesthesia, it may be accompanied by some complications, such as infection and rebleeding after surgical procedures. Surgery is usually considered when other less-invasive and equally effective procedures fail. There are some reports that surgical synovectomy after failure of intra-articular injection led to improvement [12].

In general, medical synovectomy is considered first. The term synoviorthesis (medical synovectomy) is commonly used to describe the intra-articular injection of a substance producing fibrosis of the synovium and subsynovial plexus $[13,14]$. There are two basic types of medical synovectomy: chemical or radiological. The radioisotopes commonly used for radiation synovectomy are yttrium-90 and phosphorus-32. Radiation synovectomy showed good clinical outcomes in previous studies $[15,16]$. It is advantageous as a single injection, low-factor coverage, and painless procedure. Some have argued that this procedure is safe, because lifetime cancer risk is not increased and radiation-induced malignant tumors in patients with rheumatoid arthritis treated by radiation synovectomy were absent [17-20]. However, several chromosomal studies demonstrated the possibility of transitory damage without malignant transformation [15]. Two cases of acute lymphocytic leukemia related to radiation synovectomy were reported in two children with hemophilia [21]. Moreover, the radioactive isotype is costly and not easily obtainable [13].

Rifampicin is one of the materials of chemical synovectomy that is easily available $[15,22]$. Rifampicin synovectomy showed effectiveness in previous studies [10,22-25]. With other materials, such as osmic acid, the results of synovectomy were not good as with radiation synovectomy $[16,18]$. However, rifampicin synovectomy was as effective as radiation synovectomy $[2,24]$, and does not prevent the use of either radiotherapy or surgery later [26].

From these perspectives, chemical synovectomy with rifampicin is already widely used to treat chronic hemophilic synovitis $[22,24]$. However, there are only a few reports in Asia, and our study is the first report in Korea. Despite clinical advantages, arthropathy is not completely curable, in that it may continue to degenerate. The best method to delay its progression is not clear. The aim of this study is to evaluate the effectiveness of rifampicin synovectomy in Korean hemophilia patients in many clinical aspects, including its radiologic status. In addition, we want to know whether it is still efficacious when we treat with a 2-week interval injection protocol. 


\section{MATERIALS AND METHODS}

\section{Subjects}

This was a nonrandomized, pre-post intervention study performed in the Korean Hemophilia Foundation, including hemophilic patients suffering from arthropathy. All the patient had undergone clinical evaluations based on the Fernandez-Palazzi classification. The ones with persistent synovitis and recurrent bleeding episodes that were unresponsive to factor-replacement therapies and with no history of previous chemical or surgical synovectomy on the same joint were selected. Those who had stage-IV synovitis by the Fernandez-Palazzi classification were also excluded, because previous studies had proved that synoviorthesis was not helpful and was contraindicated in this stage $[24,25]$. We performed chemical synovectomy using rifampicin at 30 joints (10 elbows, 8 knees, 12 ankles) of 28 hemophilic patients with a diagnosis of hemophilic arthropathy stage I-III.

\section{Rifampicin synovectomy}

All selected patients were under secondary prophylaxis for target joints and had received 25 units/ $\mathrm{kg}$ of antihemophilic factor (AHF) two times per week. Patients were covered with AHF concentrate up to $30 \%$ of plasma level concentration on the day of the injection and had continued infusion for 24 hours after the procedure to prevent the risk of bleeding complications $[23,25]$. A 22-gauge needle was used, and local lidocaine was injected first. We aspirated fluid before injecting the chemical substance. The dosage of rifampicin (Rifaldin; Aventis Pharma, Madrid, Spain) used for the knee was 500 mg with 7-10 mL normal saline. The knee injection is performed by a suprapatellar lateral route above the lateral corner of the patella and goes directly into the suprapatellar pouch [27]. For elbow and ankle joint injection, the entry point and joint structure were identified by ultrasonography and marked by surgical pen; the dosage of rifampicin was $250 \mathrm{mg}$ with 3-5 mL normal saline. For synoviorthesis of the elbow joint, the needle should be inserted in the center of the triangle formed by the olecranon, the radial head, and the lateral epicondyle. To perform ankle injection, the patient is positioned supine with the ankle in a neutral position to relax the anterior capsule and allow identification of the tibialis anterior tendon, which is the principal landmark. The needle should be injected into the depression between the tibialis anterior tendon medially and the tendinous expansion of the common toe extensors laterally, aiming posteriorly and slightly superior, to enter the joint just above the dome of the talus [27]. Right after the procedure, all joints were compressed for 24 hours with bandages [25,27]. Acetaminophen was administered orally when needed, but no other parenteral analgesia was used. Patients were discharged after 6 hours and encouraged to gait as early as possible. Intraarticular injections of rifampicin were performed every 2 weeks consecutively, with the total number of injections ranging from four to six $[23,26]$. Although the patients complained of pain for a week after the first injection, the pain diminished as injections were repeated and was relieved within a day after the third injection in most patients.

Clinical status (bleeding frequency, pain, joint physical status) and radiological staging were evaluated as parts of the WFH scoring system before and 1 year after the rifampicin synovectomy. Joint physical status, as mentioned above, includes swelling, range of motion, contracture, crepitus, muscle atrophy, axial deformity, and instability. The WFH pain score describes the patient's subjective joint pain as grade 0 (no pain) to grade 3 (severe). For radiologic staging, the Arnold-Hilgartner radiographic scale (AH scale) and the Pettersson score by plane X-ray, which is preferred by the WFH, were checked [28]. The $\mathrm{AH}$ scale is categorized into six stages (from stage 0 to

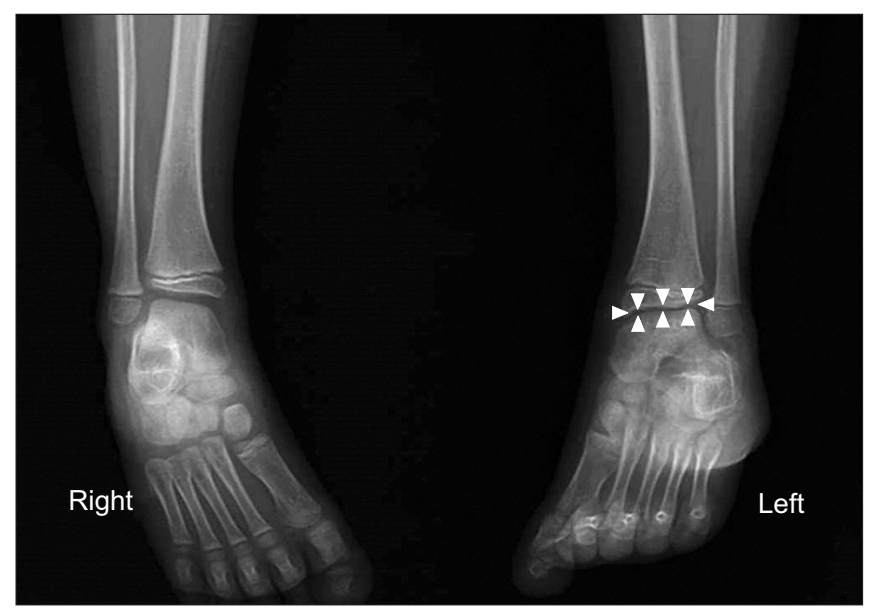

Fig. 1. Plain radiograph of a patient with hemophilic arthropathy on the left ankle. Tibiotalar joint of left ankle is narrowed, which indicates stage 4 on the Arnold-Hilgartner scale (arrowhead). 
stage 5). The presence of joint-space narrowing indicates stage 4; and absence of the narrowing means stage 3 or less [29]. Assuming that joint-space narrowing on plane radiography may affect the outcome of treatment, the patients were divided into two groups, by the AH scale of the initial X-ray, where stage 3 or less defined the lowstage group $(n=17)$, over 3 the high-stage group $(n=13)$. A case of plane X-ray with joint-space narrowing is seen in Fig. 1.

\section{Analysis}

Statistical analysis was performed using SPSS software version 17.0 (SPSS Inc., Chicago, IL, USA). A pairedsample t-test was used to compare the pre- and postchemical synovectomy means of the evaluated parameters. Data are reported as mean \pm standard deviation or proportions as appropriate. Significance was defined as $\mathrm{p}<0.05$.

\section{RESULTS}

All 30 joints of the 28 hemophilic patients completed

Table 1. Basic characteristics of participants

\begin{tabular}{|cc}
\hline \multicolumn{1}{c}{ Characteristic } & Value \\
\hline Gender, male & 28 \\
Type of haemophilia & \\
A & 25 \\
\hline B & 3 \\
\hline Age (yr) & $20.60 \pm 12.90$ \\
\hline Joint treated & \\
\hline Knee & 8 \\
\hline Ankle & 12 \\
\hline Elbow & 10 \\
\hline
\end{tabular}

Values are presented as number or mean \pm standard deviation. treatment successfully without any adverse response. All patients were men; their mean age was 20.6 years (range, 5-52 years) (Tables 1, 2). By analyzing the 30 joints of the 28 hemophilic patients, the WFH joint physical score was reduced from $9.27 \pm 3.57$ to $4.43 \pm 4.07$ ( $\mathrm{p}<0.05)$. Regardless of the type of joint, the score decreased, and the difference for each joint is shown in Fig. 2. The number of bleeding episodes were reduced from $2.07 \pm 0.78$ to $0.63 \pm 0.60$ according to the WFH system $(\mathrm{p}<0.05)$ (Fig. $3 \mathrm{~A}$ ). The pain score decreased from $2.03 \pm 0.72$ to $0.60 \pm 0.72$ $(p<0.05)$; the score for each joint is shown in Fig. 3B. For other subscores of the WFH joint physical score, only swelling, range of motion, and crepitus showed statistically significant improvement $(\mathrm{p}<0.05)$. There were no changes in contracture, muscle atrophy, deformity, or instability (Fig. 3C).

According to the severity of the radiologic finding, the WFH joint physical score of the low-stage group was reduced from $8.65 \pm 3.41$ to $3.21 \pm 3.66$, that of the high-stage group from $10.08 \pm 3.75$ to $6.04 \pm 4.15(\mathrm{p}<0.05)$ (Fig. 4A). On the other hand, the radiographic stage was unchanged

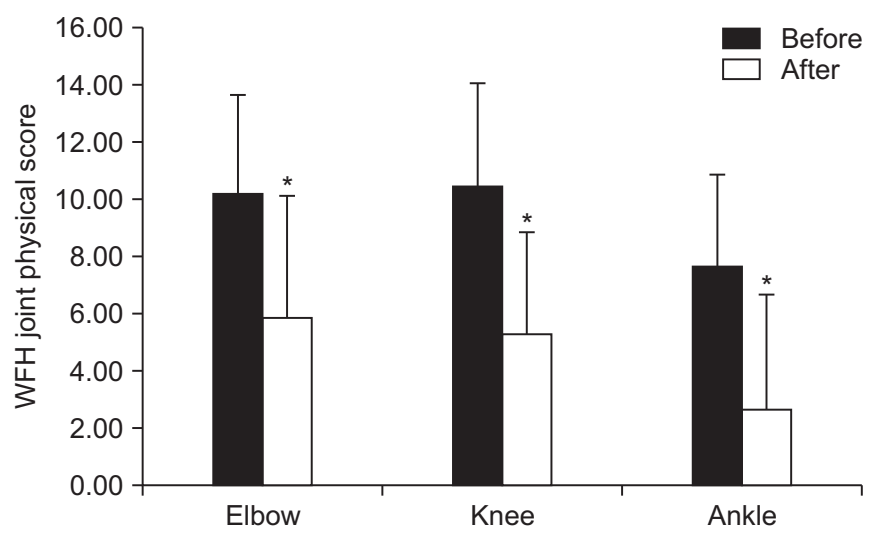

Fig. 2. World Federation of Hemophilia (WFH) joint physical score of each joint before and after rifampicin synovectomy. ${ }^{*} \mathrm{p}<0.05$ by a paired-sample t-test.

Table 2. Clinical evaluations for the 30 joints treated

\begin{tabular}{lcccc}
\hline & Knee & Ankle & Elbow & Total \\
\hline Fernandez-Palazzi clinical classification & & & & \\
I & 3 & 3 & 2 & 8 \\
\hline II & 3 & 7 & 2 & 12 \\
III & 2 & 2 & 6 & 10 \\
Pettersson score & $3.00 \pm 2.51$ & $3.08 \pm 2.23$ & $5.50 \pm 3.41$ & $3.87 \pm 2.90$ \\
Arnold-Hilgartner scale & $2.50 \pm 1.07$ & $2.79 \pm 1.03$ & $3.30 \pm 1.36$ & $2.88 \pm 1.16$ \\
\hline
\end{tabular}

Values are presented as number of joints or mean \pm standard deviation. 
(A)

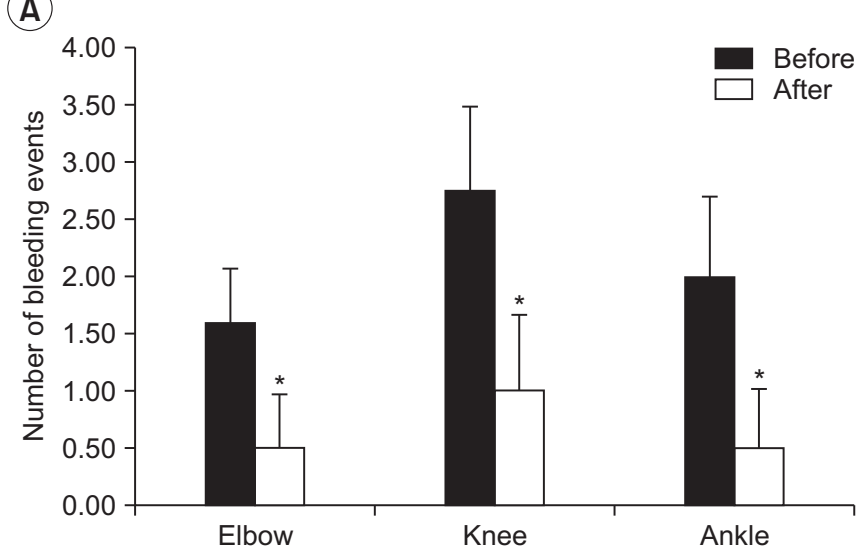

(C)

(C)

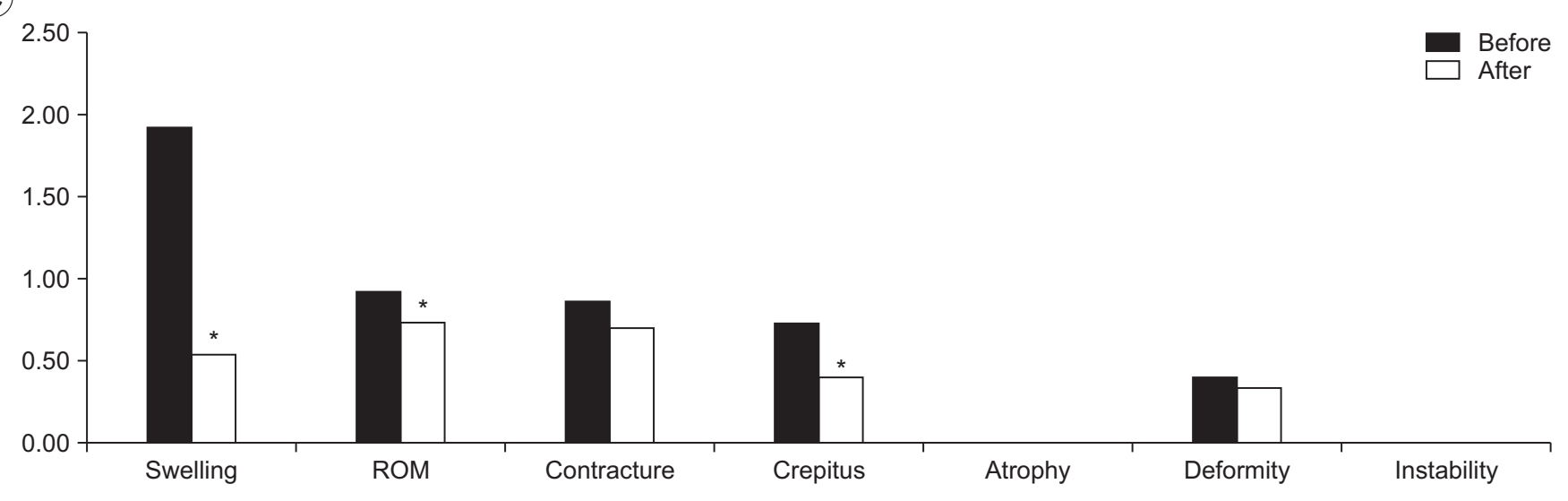

(B)

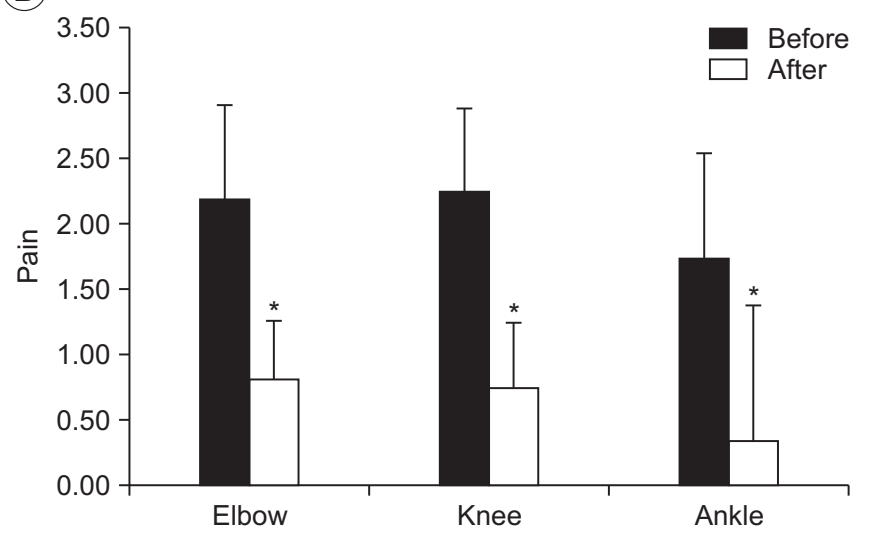

Fig. 3. Mean number of bleeding events (A) and the pain score of each joint before and after treatment (B). The rest of the subscores of the World Federation of Hemophilia (WFH) joint physical score (C). * $<<0.05$ by a paired-sample t-test.

(A)

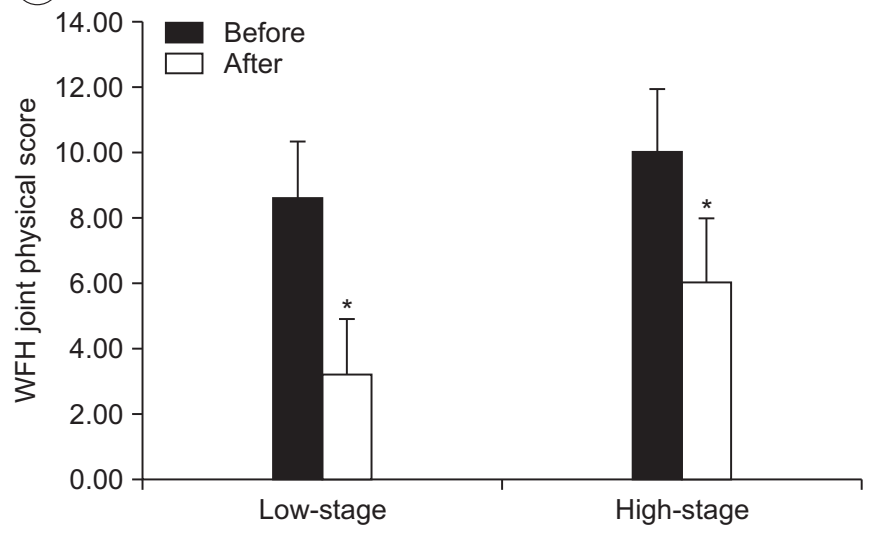




\section{DISCUSSION}

In this study, chemical synovectomy at intervals of 2 weeks using rifampicin showed a satisfactory outcome. Although previous studies were performed every week [15,22-24], we performed chemical synovectomy every 2 weeks, because the 1 -week interval was not sufficient for patients to return to normal daily activities before the next injection. Pain after the procedure induced 2-4 days immobilization and disuse of joints, which increased school and workplace absence if we injected every week. To minimize these complications, a 2-week interval was necessary, and patients showed more compliance because they could carry out daily activities and attend work or school before the next injection. However, to investigate which is more effective, the protocols of 2-week and weekly injections should be evaluated by a comparative study. To reduce post-injection pain, although intraarticular injection is widely performed by blind technique, ultrasound-guided injection delivers chemical agents more precisely to the target and may reduce the pain. Also, rifampicin with lidocaine instead of normal saline may reduce pain.

As in many studies already reported, intervention at an early stage of arthropathy shows a better outcome than at a later stage $[15,24,25]$. Although Fernandez-Palazzi [13] reported a good outcome with rifampicin synovectomy, radiologic evaluation was absent. Caviglia et al. [22] observed for 2 years, and rifampicin synovectomy showed good results, especially in the early stage of the knee joint. They suggest that young children respond well to the treatment, but this could be selection bias, in that the joints of younger children might be at a relatively earlier stage of arthropathy. Eshghi et al. [23] reported the effectiveness of rifampicin synovectomy in an Asian population, but there was no study comparing the early and later stages.

The most important points of this study are that we not only proved the effectiveness of rifampicin synovectomy on Korean hemophilia, but also suggested radiological criteria for the early stage. In addition, the subjects' ages in this study were relatively higher than in previous studies, but the result was comparable or better. In Korea, because of preventive treatment from childhood to reduce the frequency of bleeding in joints, the patients with arthropathy were more commonly adolescents rather than young children.

Rodriguez-Merchan et al. [15] compared rifampicin synovectomy and radiation synovectomy with yttrium-90 and found that the early-stage group by the Pettersson score responded better to treatment than the later-stage group did. They recommended the use of rifampicin in elbow and ankle, but preferred yttrium-90 for the knee. In this report, the authors divided clinical status with the Pettersson score into 5 stages, but the categorization lacked radiologic criteria. In our study, we did not compare rifampicin synovectomy with other treatments, but suggest detailed criteria for the early stage in terms of radiology.

Plain radiography remains the standard method of joint evaluation in hemophilic arthropathy. There have been various classifications of hemophilic arthropathy, notably the AH scale developed in the United States, and the Pettersson score, which is preferred by the WFH [28]. Although there are many reports of the advantages of rifampicin synovectomy in the early stage, there is no definite criteria that suggests radiologically early stage yet. Some report that the treatment failed in joints with a narrow joint space, such as the elbow [10], and given that a narrowed joint space may suggest significant cartilage damage and disturb the distribution of the chemical agent, we assumed that the joint space narrowing should be considered in evaluating radiological status. Jointspace narrowing was mentioned in stage 4 of the $\mathrm{AH}$ scale, which may be more applicable than the Pettersson score in this aspect [29].

There are several limitations of this study. First, it was a short-period study, in that we compared the effectiveness of rifampicin synovectomy for 1 year after treatment. To date we cannot say if our treatment program delays functional impairment in the long term, because of the lack of a longer follow-up. Hemophilic arthropathy is a lifelong disease on which studies should focus on the long-term outcome. The efficacy of chemical synovectomy with osmic acid and radiation synovectomy with yttrium-90 has persisted for up to 9 years in one group of patients [16]. Rifampicin is the most commonly used material for chemical synovectomy; more reports should be accumulated on it. We plan to follow up the patients every year in the future.

Although plain radiograph is the gold-standard tool for hemophilic arthropathy, there are limitations, in 
that soft-tissue lesions and synovial proliferation are not recognizable. Ultrasonography is ideal for finding the presence, extent, and indeed the nature of soft-tissue swellings. This is particularly suitable for patients with hemophilic arthropathy, because the early stages of the disease are largely confined to the synovium [28]. Ultrasound can detect the synovial involvement accurately, and staging of ultrasonographic findings may reflect a more exact status of arthropathy [30]. It is also noninvasive, inexpensive, and readily available, with no reported complications. To detect an early stage of synovial or cartilage alteration, magnetic resonance imaging (MRI) also might be helpful, in that it shows everything, including bone and soft tissues [31]. Although there are some investigations to develop a semiquantative system for assessing hemophilic arthropathy by MRI [32], one of the most decisive disadvantage is its high cost. In this perspective, we could have suggested more information about the early stage of radiological evaluation if we had performed an ultrasonography work-up. Further studies of rifampicin synovectomy with MRI and ultrasonography findings with clinical assessment of hemophilic arthropathy may present more criteria for early-stage intervention.

In conclusion, rifampicin synovectomy seems to improve the clinical status of patients with hemophilia in Korea. Compared to the previous literature, our findings reconfirm that chemical synovectomy using rifampicin is a cost-effective, simple, and practical method in the management of hemophilic arthropathy $[10,24,25,32]$. Although the mechanism of rifampicin on an arthropathic joint is not clearly elucidated, considering its effectiveness in the early stage of arthropathy, it presumably inhibits inflammation and hypertrophy of the synovium, which reduces bleeding episodes and delays progression to joint degeneration and destruction $[4,5]$. Furthermore, molecular biologic and pathophysiologic study based on rifampicin is necessary to investigate the precise mechanism that delays the progression of joint degeneration. Arthropathic joints of a hemophilic patient may continue to degenerate for all of his or her life, so it is essential to preserve joint status intact as long as possible. Early diagnosis and proper treatment is fundamental to prevent disability. From these results and perspectives, to reduce the complications and to maintain the physiological function of joints, rifampicin synovectomy in an early stage of hemophilic arthropathy should be considered as a treatment option.

\section{CONFLICT OF INTEREST}

No potential conflict of interest relevant to this article was reported.

\section{REFERENCES}

1. Vucic M, Draskovic D. Frequency and degree of chronic arthropathy in hemophilia a patients on prophylactic and on-demand treatment. Acta Medica Medianae 2016;55:38-43.

2. Rodriguez-Merchan EC. Methods to treat chronic haemophilic synovitis. Haemophilia 2001;7:1-5.

3. Rodriguez-Merchan EC. Haemophilic synovitis: basic concepts. Haemophilia 2007;13 Suppl 3:1-3.

4. Hoots WK, Rodriguez N, Boggio L, Valentino LA. Pathogenesis of haemophilic synovitis: clinical aspects. Haemophilia 2007;13 Suppl 3:4-9.

5. Rodriguez-Merchan EC, Wiedel JD. General principles and indications of synoviorthesis (medical synovectomy) in haemophilia. Haemophilia 2001;7 Suppl 2:6-10.

6. Manco-Johnson MJ, Abshire TC, Shapiro AD, Riske B, Hacker MR, Kilcoyne R, et al. Prophylaxis versus episodic treatment to prevent joint disease in boys with severe hemophilia. N Engl J Med 2007;357:535-44.

7. Berntorp E, Astermark J, Bjorkman S, Blanchette VS, Fischer K, Giangrande PL, et al. Consensus perspectives on prophylactic therapy for haemophilia: summary statement. Haemophilia 2003;9 Suppl 1:1-4.

8. Lee CA. Prevention of haemophilic synovitis: prophylaxis. Haemophilia 2007;13 Suppl 3:20-5.

9. Zengin O, Okan V, Ozkur A, Sayiner ZA, Sahin HH, Yilmaz M, et al. The relationship of frequency and intensity of bleeding with hemophilic arthropathy in hemophilia patients, radiological assessment and socio-economic impacts of the arthropathy. UHOD 2015;25:32-7.

10. Radossi P, Baggio R, Petris U, De Biasi E, Risato R, Davoli PG, et al. Intra-articular rifamycin in haemophilic arthropathy. Haemophilia 2003;9:60-3.

11. Llinas A. The role of synovectomy in the management of a target joint. Haemophilia 2008;14 Suppl 3:177-80.

12. Combe B, Krause E, Sany J. Treatment of chronic knee 
synovitis with arthroscopic synovectomy after failure of intraarticular injection of radionuclide. Arthritis Rheum 1989;32:10-4.

13. Fernandez-Palazzi F. Treatment of acute and chronic synovitis by non-surgical means. Haemophilia 1998;4:518-23.

14. Pasta G, Mancuso ME, Perfetto OS, Solimeno LP. Synoviorthesis in haemophilia patients with inhibitors. Haemophilia 2008;14 Suppl 6:52-5.

15. Rodriguez-Merchan EC, Caviglia HA, Magallon M, Perez-Bianco R. Chemical synovectomy vs. radioactive synovectomy for the treatment of chronic haemophilic synovitis: a prospective short-term study. Haemophilia 1997;3:118-22.

16. Molho P, Verrier P, Stieltjes N, Schacher JM, Ounnoughene $\mathrm{N}$, Vassilieff $\mathrm{D}$, et al. A retrospective study on chemical and radioactive synovectomy in severe haemophilia patients with recurrent haemarthrosis. Haemophilia 1999;5:115-23.

17. Silva M. Luck Jr JV, Llinas A. Chronic hemophilic synovitis: the role of radiosynovectomy. Treat Hemophilia 2004;33:1-10.

18. Rivard GE. Chemical synovectomy in haemophilia: status and challenges. Haemophilia 2001;7 Suppl 2:16-9.

19. Rodriguez-Merchan EC, Valentino LA. Safety of radiation exposure after radiosynovectomy in paediatric patients with haemophilia. Haemophilia 2015;21:4118.

20. Gilbert MS, Cornwall R. The history of synoviorthesis in haemophilia. Haemophilia 2001;7 Suppl 2:3-5.

21. Dunn AL, Manco-Johnson M, Busch MT, Balark KL, Abshire TC. Leukemia and P32 radionuclide synovectomy for hemophilic arthropathy. J Thromb Haemost 2005;3:1541-2.
22. Caviglia H, Galatro G, Duhalde C, Perez-Bianco R. Haemophilic synovitis: is rifampicin an alternative? Haemophilia 1998;4:514-7.

23. Eshghi P, Goudarzipour K, Davoudabadi FH, Aghakhani R. Intra articular injection of rifampin in Iranian children with haemophilic arthropathy. Iran J Blood Cancer 2010;2:123-7.

24. Fernandez-Palazzi F, Rivas S, Viso R, de Bosch NB, de Saez AR, Boadas A. Synovectomy with rifampicine in haemophilic haemarthrosis. Haemophilia 2000;6:5625.

25. Rezazadeh S, Haghighat A, Mahmoodi M, Babanezhad Z, Karimi M. Synoviorthesis induced by rifampicin in hemophilic arthropathy: a report of 24 treated joints. Ann Hematol 2011;90:963-9.

26. Battistella LR. Maintenance of musculoskeletal function in people with haemophilia. Haemophilia 1998;4 Suppl 2:26-32.

27. Rodriguez-Merchan EC, Goddard NJ. The technique of synoviorthesis. Haemophilia 2001;7 Suppl 2:11-5.

28. Goddard NJ, Mann H. Diagnosis of haemophilic synovitis. Haemophilia 2007;13 Suppl 3:14-9.

29. Arnold WD, Hilgartner MW. Hemophilic arthropathy: current concepts of pathogenesis and management. J Bone Joint Surg Am 1977;59:287-305.

30. Zukotynski K, Jarrin J, Babyn PS, Carcao M, PazminoCanizares J, Stain AM, et al. Sonography for assessment of haemophilic arthropathy in children: a systematic protocol. Haemophilia 2007;13:293-304.

31. Soler R, Lopez-Fernandez F, Rodriguez E, Marini M. Hemophilic arthropathy: a scoring system for magnetic resonance imaging. Eur Radiol 2002;12:836-43.

32. Caviglia HA, Fernandez-Palazzi F, Galatro G, PerezBianco R. Chemical synoviorthesis with rifampicin in haemophilia. Haemophilia 2001;7 Suppl 2:26-30. 